\title{
A Novel Reagent for tert-Butoxycarbonylation of Amino Acids
}

Sythesis of 1-tert-butoxy-2-tert-butoxycarbonyl-1,2-dihydroisoquinoline (BBDI):<smiles>c1ccc2cnccc2c1</smiles>

(1.0 equiv)

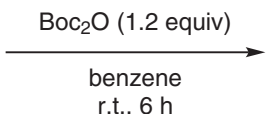

r.t., $6 \mathrm{~h}$

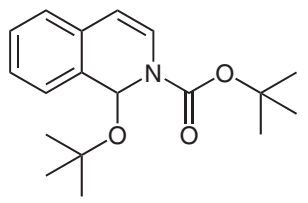

BBDI

$92 \%$ yield

Category

Peptide Chemistry

\section{Key words}

butoxycarbonylation

amino acids

chemoselectivity

isoquinolines

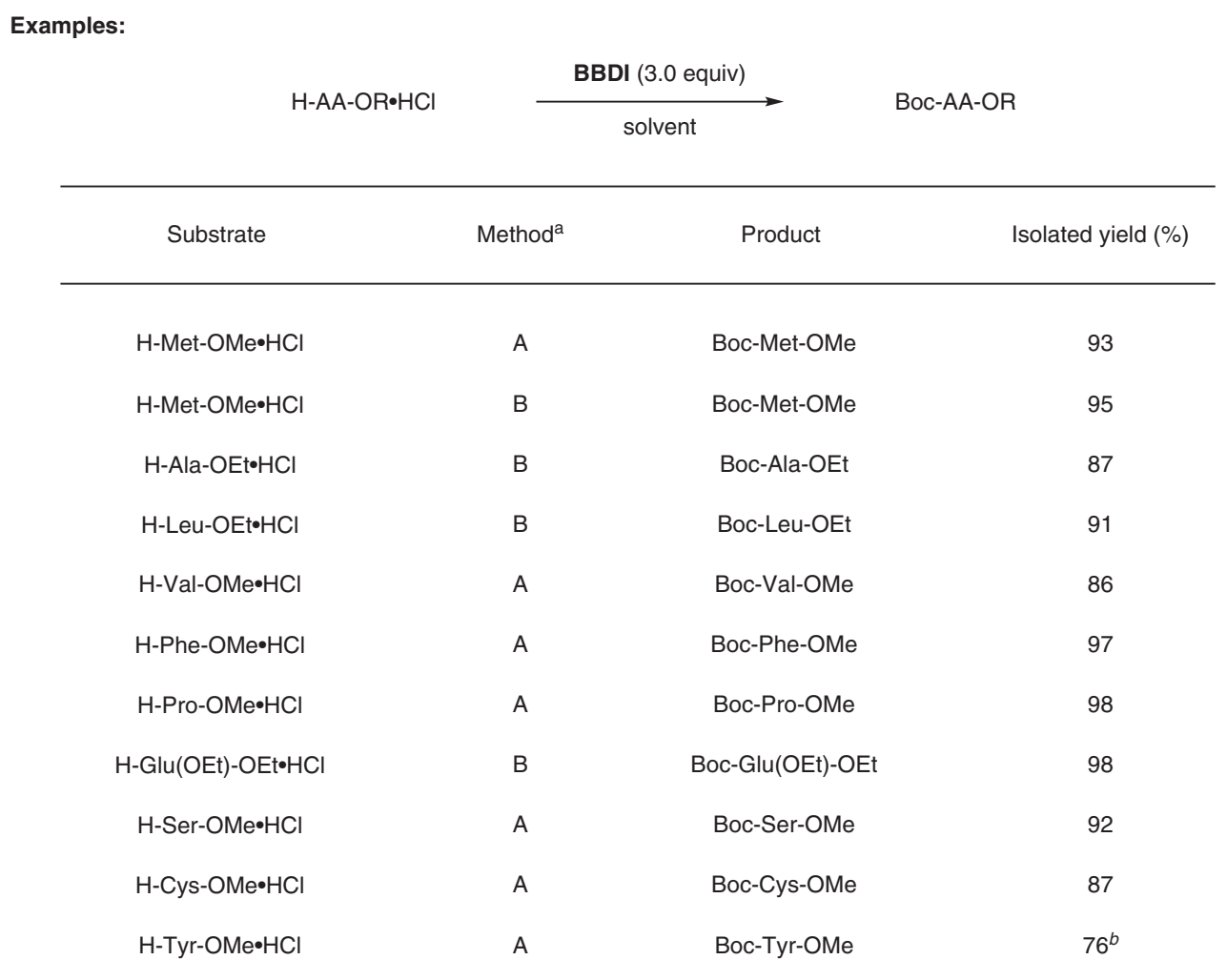

a Method A: 1,2-dimethoxyethane, r.t., overnight; method B: $\mathrm{Et}_{2} \mathrm{O}$, reflux, overnight. ${ }^{\mathrm{b}}$ Small amount of BocTyr(Boc)-OMe was also obtained.

Significance: Boc-protected amino acids are important because of their good resistance in peptide synthesis. The authors developed (BBDI) as an alternative to the conventional tert-butoxycarbonylating reagent di-tert-butyl dicarbonate for the synthesis of Boc-protected $\alpha$-amino acids.
Comment: With the tert-butoxycarbonylating reagent BBDI, various Boc-protected $\alpha$-amino acids can be synthesized from amino acid ester hydrochlorides in excellent yields. 